\title{
Legitimierung, oder das demokratische Dilemma der Euro-Rettungspolitik
}

Im Mai 2007, also vor Beginn der derzeitigen Krise, hatten $57 \%$ der Europäer in Eurobarometer-Umfragen „Vertrauen in die Europäische Union" bekannt - und im Mai 2013 waren es nur noch $31 \%$. In den Krisenländern der Währungsunion war der Rückgang besonders stark (in Spanien von $65 \%$ auf $17 \%$ ), aber auch in Deutschland halbierte sich das Vertrauen in die EU von 56\% auf 29\%. Zugleich fiel das Vertrauen in die eigene nationale Regierung im EU-Durchschnitt von $41 \%$ auf $26 \%$. Auch dabei war der Verlust in den Krisenländern besonders dramatisch - in Griechenland von $41 \%$ auf 9\% und in Spanien sogar von $52 \%$ auf $8 \%$. In Deutschland dagegen blieb der moderate Rückgang des Vertrauens in die eigene Regierung (von $49 \%$ auf $44 \%$ ) in der üblichen Schwankungsbreite demokratischer Politik.

Was besagen diese Eurobarometer-Daten über den Zustand der demokratischen Legitimität der Politik in der Union und ihren Mitgliedstaaten? Um sie zu interpretieren, bedarf es einer begrifflichen Klärung, für die man sich auf Abraham Lincolns Trias des "government of the people, by the people and for the people" beziehen kann. "Government of the people" setzt ein politisches Gemeinwesen voraus, dessen Mitglieder willens und fähig sind, sich gemeinsam selbst zu regieren - was für die Angehörigen der Europäische Union nicht einfach unterstellt werden kann.

Beim "government for the people" geht es um die fundamentale Rechtfertigung jeder politischen Herrschaft. Diese ist notwendig, um Ziele zu erreichen und Probleme zu bewältigen, die weder von autonom handelnden Einzelnen noch durch freiwillige Kooperation in der Zivilgesellschaft oder durch marktförmigen Austausch erreicht oder bewältigt werden können. Aber die Zwangsgewalt der Herrschaft und die Zumutung des Gehorsams sind nur durch die Gemeinwohlfunktion zu rechtfertigen, welche die Regierenden - im Sinne des Amtseids des Grundgesetzes - verpflichtet den Nutzen des Volkes zu mehren, Schaden von ihm abzuwenden und Gerechtigkeit gegen

\footnotetext{
Der Text stützt sich teilweise auf Argumente aus F. W. Scharpf: Eurorettung als Demokratieproblem, in: dms - der moderne staat, 6 . Jg. (2013), Nr. 2, S. 279-288.
}

jedermann zu üben. Wenn es an diesen output-orientierten Anforderungen eklatant scheitert, kann jedes Regime seine Legitimität verlieren.

Die Anforderungen des "government by the people“ gehen jedoch weiter. Das input-orientierte Legitimationsprinzip einer demokratischen Selbstregierung des Volkes impliziert, dass die Regierten zugleich die Autoren der Gesetze sein sollen, denen sie gehorchen müssen. Auch wo direkt-demokratische Entscheidungen nicht möglich sind, soll sich die Ausübung der Regierungsgewalt an den realen Interessen und Präferenzen der Regierten orientieren müssen. Gesetze sollen aus der freien politischen Diskussion im öffentlichen Raum und im Parlament hervorgehen, und zugleich soll die Abhängigkeit der Regierenden vom Ausgang allgemeiner, gleicher und periodischer Wahlen auch die antizipierende Rücksichtnahme auf die Belange nicht organisierter und wenig artikulationsfähiger Gruppen sicherstellen. Zur Herstellung input-orientierter demokratischer Legitimität genügt also nicht die Konstruktion juristischer „Legitimationsketten", die die Ausübung von Herrschaftsgewalt schließlich auf einen (fiktiven) „pouvoir constituant" zurückführen sollen. Legitimationsbegründend ist nur die reale Abhängigkeit der Regierenden vom periodischen Votum der Regierten.

In der Theorie des demokratischen Verfassungsstaates geht es also - wenn der Zusammenhalt des Gemeinwesens gesichert ist - immer zugleich um input- und outputorientierte Anforderungen, um „responsive“ und „responsible government". Und es geht darum, das Spannungsverhältnis zwischen der gleichen Selbstregierung aller Bürger und der unaufhebbaren Bindung der Herrschaftsgewalt an Gemeinwohl und Gerechtigkeit als legitimationssichernde Wechselbeziehung zu institutionalisieren. Die demokratisch legitimierte Staatsgewalt soll tatkräftig regieren können. Aber zugleich soll die Verfassung die „Tyrannei der Mehrheit“ verhindern, während Parteienkonkurrenz, öffentliche Diskussion und egalitäre Wahlen die Verselbständigung der Regierungsgewalt ausschlieBen sollen.

Die Institutionen demokratischer Verfassungsstaaten unterscheiden sich durchaus in ihrer relativen Gewich- 
Prof. Dr. Fritz W. Scharpf ist Direktor emeritus des Max-Planck-Instituts für Wirtschaftsforschung in Köln.
So lassen sich nun auch die eingangs zitierten Umfrage-Ergebnisse interpretieren. Das hohe Vertrauen in die Europäische Union vor 2007 reflektierte output-orientierte Zufriedenheit mit dem Zustand der Union, während der Mangel an input-orientierten Einflusschancen nur in der stetig fallenden Beteiligung an den Wahlen zum Europäischen Parlament zum Ausdruck kommt. Politische Forderungen und Kritik wurden stattdessen an die nationalen Regierungen adressiert und erklären so deren im Vergleich zur EU deutlich niedrigere Vertrauenswerte.

gleich mit innen kennzeichnet die Europäische Union ein ganz einseitiger Verlass auf output-orientierte Legitimationsargumente: Die Union sichert den Frieden zwischen Völkern; sie fördert den Wohlstand durch wirtschaftliche Verflechtung; und Fortschritte der Integration dienen der immer engeren Verbindung der europäischen Völker. Dagegen stand die input-orientierte Legitimation der EU immer auf schwachen Füßen: Gestützt auf die breite und unbefragte Zustimmung zur Integration an sich war europäisches Regieren - wenn man von Großbritannien und den skandinavischen Staaten absieht - lange vor politischen Kontroversen geschützt. Seine Inhalte wurden in starkem Maße durch eine unpolitische „Integration durch Recht" geprägt, d.h. durch richterliche Rechtsschöpfung und Entscheidungen der Kommission. Die europäische Gesetzgebung dagegen war auf den breiten Konsens der Regierungen im Rat angewiesen. Dazu ist heute zwar in den meisten Fällen auch die Zustimmung einer Mehrheit im Europäischen Parlament erforderlich. Aber obwohl das Parlament seine Vetoposition in der Gesetzgebung fleißig nutzt, kann in der EU von einer politischen Abhängigkeit der Regierenden vom Ausgang der Europawahl bisher keine Rede sein.

\section{Mangelnde demokratische Legitimität der Euro- Rettungspolitik}

Dennoch war das offenbare europäische Demokratiedefizit bis vor wenigen Jahren für die Bürger kaum von Bedeutung. Die Herrschaftsgewalt der Union trat innen ja nicht unmittelbar gegenüber, sondern immer vermittelt durch Akte der nationalen Gesetzgebung, Verwaltung und Rechtsprechung. Während die europäische Politik für sie unsichtbar und ungreifbar blieb, hatten die Bürger es immer mit nationalen Instanzen zu tun, und solange diese die europäischen Vorgaben loyal und stillschweigend umsetzten, profitierte die Union von einer „Legitimationsvermittlung“: Für die Input-Legitimation der vom europäischen Recht überformten nationalen Politik reichte es, dass die damit unzufriedenen Wähler die eigene Regierung zur Rechenschaft ziehen und bestrafen konnten.
In der im Herbst 2008 beginnenden internationalen Finanzkrise und der darauf folgenden Eurokrise hat jedoch die output-orientierte Zufriedenheit mit dem allgemeinen Zustand der Union nicht nur in den Krisenländern, sondern auch in bisher von der Krise verschonten Ländern wie Deutschland dramatisch abgenommen. Vor allem aber kann die mangelnde Input-Legitimation nun nicht mehr durch die demokratische Legitimation der nationalen Politik ersetzt oder verdeckt werden. In der Eurokrise musste die europäische Politik ihre Deckung verlassen. Sie hat im vollen Licht der öffentlichen Aufmerksamkeit direkt in die Lebensumstände von Millionen europäischer Bürger eingegriffen. Zugleich hat sie die input-orientierte Verantwortlichkeit der nationalen Politik sichtbar außer Kraft gesetzt. In den Schuldnerstaaten hat sie dafür gesorgt, dass auch die Abwahl von Regierungen an den von der Troika oktroyierten Maßnahmen nichts ändern konnte. Und selbst wenn der Deutsche Bundestag unter dem Druck des Verfassungsgerichts es einmal wagte, einen Beschluss der Eurogruppe über ein Wochenende aufzuhalten, so änderte das doch nichts daran, dass dieser danach von den Parlamenten aller Gläubigerstaaten unverändert beschlossen werden musste.

Kurz: Wie nie zuvor übt nun die europäische Politik, für alle sichtbar und mit unmittelbarer Wirkung, politische Herrschaftsgewalt aus. Die Frage nach ihrer input-orientierten Legitimität muss jetzt also auf der europäischen Ebene gestellt und beantwortet werden.

\section{Fremdherrschaft oder autoritäres Expertenregime}

Dort freilich sind die Institutionen und Prozesse, in denen über die Europolitik entschieden wird, in perfekter Weise gegen die Einflüsse demokratischer Inputs abgeschirmt. Dies gilt offensichtlich für die Europäische Zentralbank, die mit strikt begrenztem Mandat jeder direkten oder indirekten demokratischen Verantwortlichkeit entzogen ist. Trotzdem wurde sie zum entscheidenden Akteur der Euro-Rettung, von dessen Politik es nicht nur abhängt, ob die Stagnation der Eurozone in die Depression oder in eskalierende Inflation führt, sondern auch, ob ein Mit- 
gliedstaat wie Zypern dem sofortigen Bankrott überlassen oder noch in letzter Minute gerettet wird.

Ähnliches gilt für die Europäische Kommission, deren Autorität als neutrale Hüterin des europäischen Gemeinwohls gerade nicht auf demokratischer Verantwortlichkeit beruht. Aber sie ist es, die die „Memoranda of Understanding" definiert, die demokratisch gewählte Regierungen unterschreiben mussten, um die jeweils nächste Tranche der europäischen Rettungskredite zu erhalten. Und noch stärker ist ihre politische Rolle in dem neuen Euro-Regime der Six-Pack- und Two-PackVerordnungen ausgestaltet. Im Rahmen des Verfahrens zur „Korrektur makroökonomischer Ungleichgewichte“ können Empfehlungen, die allein von ihrer ökonomischen Einschätzung abhängen, und die sich auf sämtliche Kompetenzbereiche eines Mitgliedstaates erstrecken und mit harten Sanktionen durchgesetzt werden können, vom Rat der Eurogruppe allenfalls mit „umgekehrt qualifizierter Mehrheit" modifiziert oder blockiert werden.

Aber selbst wenn dafür ein positives Votum des Rats nötig wäre, könnte dieses eine Input-Legitimation der im Zuge der Euro-Rettung durchzusetzenden Maßnahmen nicht begründen. Die Vertreter der nationalen Regierungen sind durch ihre jeweiligen Parlamente und Wähler allenfalls indirekt-demokratisch dazu legitimiert, Verpflichtungen für das eigene Land einzugehen und allgemeinen Regeln für alle Mitgliedstaaten zuzustimmen. Aber die deutschen Wähler und der Deutsche Bundestag könnten den Bundesfinanzminister und die Kanzlerin nicht zu diskretionären Einzelfall-Entscheidungen legitimieren, die den Bürgern Portugals schwerste Opfer auferlegen. Aus deren Perspektive jedenfalls handelt es sich dabei um die demokratisch nicht legitimierbare Herrschaft fremder Regierungen. Und daran würde sich im Prinzip auch dann nichts ändern, wenn Einzelentscheidungen durch das Europäische Parlament gebilligt werden müssten.

Der steile Abfall des EU-Vertrauens kann also auch der Frustration der Bürger geschuldet sein, die nun nicht nur die Macht der europäischen Politik, sondern auch die eigene politische Hilflosigkeit ihr gegenüber erkannt haben. Und ebenso reflektiert der extreme Verlust von Vertrauen in die nationalen Regierungen der Krisenstaaten nicht nur die output-orientierte Verzweiflung über wirtschaftlichen Niedergang und soziale Verelendung, sondern auch den Zorn über die Blockade input-orientierter Einflussmöglichkeiten auf die Richtung der nationalen Politik. Kurz: Gemessen an den Anforderungen demokratischer Legitimität erscheint die bisherige EuroRettungspolitik als eine Katastrophe. Und gemessen an ihren institutionellen Grundlagen kann sie nur als Fremdherrschaft kombiniert mit einem autoritären Expertenregime qualifiziert werden.

Solange der unmittelbare Kollaps des Euro drohte, konnte man sich dafür vielleicht auf „übergesetzlichen Notstand“ berufen. Aber inzwischen wachsen offenbar selbst in der europäischen Politik die Zweifel an der politischen Akzeptanz des gegenwärtigen Euro-Regimes. Während die EZB lediglich einen „europäischen Finanzminister“ für notwendig hielt, denkt man in Brüssel und Berlin sogar daran, entweder den Präsidenten der Kommission oder einen der Ratspräsidenten europaweit wählen zu lassen. Damit soll freilich in erster Linie die Autorität des Regimes gestärkt werden, während man die für richtig gehaltenen Maßnahmen der bisherigen Europolitik keineswegs zur demokratischen Disposition stellen möchte. Es geht hier also vor allem um den Anschein von Demokratie - nach dem aus Westernfilmen bekannten Argument zur Verhinderung von Lynchjustiz: „Let's give him a fair trial and then hang him". Aber ließe sich das ändern?

\section{Politisierung der Euro-Rettungspolitik als Weg zur Demokratisierung?}

Könnte etwa die Bewältigung der Eurokrise auch aus politischen Prozessen hervorgehen, die input-orientierte demokratische Legitimität auf der europäischen Ebene vermitteln können? Jürgen Habermas und andere pro-europäische Demokraten scheinen das für möglich zu halten. Und die Absicht der Partei-Familien im Europäischen Parlament, die kommende Wahl als europaweite Konkurrenz ihrer Spitzenkandidaten um die Position des Kommissionspräsidenten zu inszenieren, scheint einen möglichen Weg zu diesem Ziel zu eröffnen. In der Tat könnten auch die pro-europäischen Parteien, wenn sie mit einer in allen Euroländern einheitlichen Position den Wahlkampf führen wollten, die politische Auseinandersetzung über die Optionen der Euro-Krisenpolitik nicht länger so vermeiden, wie sie das in der letzten Bundestagswahl getan haben. Und wenn das Wahlergebnis dann die Auswahl des Präsidenten der EU-Kommission bestimmte, dann hätten die europäischen Wähler in der Tat, und zum ersten Mal, auch einen gewissen Einfluss auf die Richtung der europäischen Politik erreicht.

Dies sind viele Konditionalsätze, und die Multiplikation der Wahrscheinlichkeiten wird wohl nicht gegen eins konvergieren. Trotzdem lohnt es sich, die Implikationen einer europaweiten oder jedenfalls eurozonen-weiten Politisierung der Euro-Rettungspolitik in Betracht zu ziehen. Damit stellen sich zwei Fragen: Was wären die Optionen, über die politisch gestritten werden müsste? Und 
welche Rückwirkung hätte dieser politische Streit auf die Legitimität der europäischen Politik? Aber um die wählbaren Optionen zu identifizieren, müssen zunächst die in Deutschland überwiegend falsch dargestellten Ursachen der Eurokrise richtig verstanden werden. Da ich mich dazu wiederholt geäußert habe, ${ }^{1}$ will ich mich hier auf knappe Thesen beschränken.

\section{Ursachen der Eurokrise}

Die Währungsunion umfasste und umfasst heterogene Ökonomien mit unterschiedlicher Wirtschaftsstruktur, unterschiedlichen Lohnbildungssystemen und deshalb auch unterschiedlicher Inflationsdynamik (die früheren „Hartwährungsländer“ und „Weichwährungsländer“). Mit dem Eintritt in die Währungsunion entfiel für die Eurostaaten die Notwendigkeit, ihre Zahlungsbilanz auszugleichen, und sie verloren die Möglichkeit, die Gesamtnachfrage mit den Mitteln der Währungs- und Geldpolitik zu beeinflussen. Zugleich wurde die Nutzung der Fiskalpolitik zur Bekämpfung einer Rezession durch den Stabilitätspakt beschränkt.

Die stabilitätsorientierte Geld- und Währungspolitik der EZB musste sich jedoch am Durchschnitt der Eurozone orientieren. Sie war also für die Hartwährungsländer zu restriktiv und für die früheren Weichwährungsländer zu expansiv. Sie hat deshalb die Binnennachfrage und damit auch die Importe in den einen (darunter in Deutschland) übermäßig reduziert, in den anderen (darunter Griechenland, Irland, Portugal und Spanien - den GIPS-Ländern) übermäßig stimuliert.

Im Ergebnis kam es deshalb in Deutschland nach 2001 zu einer langen Rezession mit steigender Arbeitslosigkeit, stagnierenden Löhnen und rasch zunehmenden Leistungsbilanzüberschüssen. In den GIPS-Ländern dagegen erzeugte das zu billige Geld eine kreditfinanzierte Überkonjunktur mit steigender Beschäftigung, steigenden Löhnen und steigenden Leistungsbilanzdefiziten. Ein weiteres Ergebnis war die Verzerrung der realen Wechselkurse, so dass die Exporte der Überschussländer von einer realen Unterbewertung profitierten, während die Wirt-

1 F. W. Scharpf: Monetary Union, Fiscal Crisis, and the Pre-emption of Democracy, in: Zeitschrift für Staats- und Europawissenschaften, 9. Jg. (2011), Nr. 2, S. 163-168; ders.: Legitimacy Intermediation in the Multilevel European Polity and its Collapse in the Euro Crisis, MPIfG Discussion Paper, Nr. 12/16, Köln 2012, Max Planck Institute for the Study of Societies. Jetzt in: K. Armingean (Hrsg.): Staatstätigkeiten, Parteien, Demokratie, Festschrift für Manfred G. Schmidt, S. 567596; ders.: Political Legitimacy in a Non-optimal Currency Area, MPIfG Discussion Paper, Nr. 13/15, 2013, http://www.mpi-fg-koeln. mpg.de/pu/mpifg_dp/dp13-15.pdf. schaft der Defizitländer durch eine starke Überbewertung belastet wurde.

Zwischen 1999 und 2008 wurden die wachsenden GIPSDefizite durch den Zufluss privaten Kapitals aus den Überschussländern finanziert. Dieser ökonomisch schein-rationale Zirkel wurde durch die internationale Finanzkrise im Herbst 2008 unterbrochen, die eine weltweite Kreditklemme auslöste. In den vom Zufluss ausländischen Kapitals abhängigen Defizitländern war die Folge eine besonders tiefe Wirtschafts- und Bankenkrise, die dann (und erst dann!) auch die Staatsverschuldung steil ansteigen ließ. Auf diese reagierten „die Märkte“ bei Ländern mit hohen Leistungsbilanzdefiziten (und nur bei solchen!) schließlich mit so hohen Risikozinsen, dass im Frühjahr 2010 in Griechenland und dann auch in Irland und Portugal die Staatsinsolvenz drohte, die auch den Verbleib der betroffenen Länder in der Währungsunion hätte in Frage stellen können.

Und weil bei einem Kollaps der Schuldnerstaaten auch die zuvor aufgetürmten Gläubigerpositionen und Exportvorteile der Überschussländer hätten kollabieren können, entschied man sich im Frühjahr 2010 in Brüssel, Frankfurt, Paris und Berlin zur „Euro-Rettung um jeden Preis“.

\section{Optionen der Euro-Rettung}

Wenn die Währungsunion selbst nicht in Frage gestellt werden durfte, dann musste es in der Eurokrise um die Bewältigung der folgenden Probleme gehen:

- die Verhinderung der Insolvenz akut gefährdeter Staaten,

- den Abbau bestehender Leistungsbilanzungleichgewichte und die Korrektur der wettbewerbsverzerrenden realen Wechselkurse,

- die Überwindung der als Folge der Eurokrise entstandenen Wirtschaftskrisen in den Defizitländern.

- Und gerade wenn die Rettung „um jeden Preis“ erfolgen sollte, musste es dabei auch um die faire Verteilung der Vorteile und Kosten gehen.

Dies sind extrem anspruchsvolle und zum Teil miteinander unvereinbare Ziele, die nicht mit gleicher Priorität angegangen werden konnten. In der Praxis hat bisher die von Deutschland favorisierte Austeritäts- und Reformpolitik dominiert, die sich auf die ersten beiden Aufgaben konzentrierte und die beiden anderen ignorierte. Sie 
trifft inzwischen auf zunehmende Kritik, die in der Konsequenz auf die Alternativoption einer Transferunion zielt, ohne dass diese Implikation in der politischen Diskussion schon deutlich wäre.

\section{Austerität und Strukturreformen}

Am Beginn der Eurokrise ging es nur um die erste der genannten Anforderungen: Akut drohende Insolvenzen wurden zunächst durch Kredite der Rettungsfonds vermieden. Einen deutlichen Rückgang der Risikozinsen erzielte jedoch erst die Geldpolitik der EZB mit der (in ihrer Legalität umstrittenen) Ankündigung, sie werde notfalls zur Rettung des Euro auch Anleihen gefährdeter Staaten unmittelbar aufkaufen. Bisher hat diese Ankündigung ausgereicht, um die Gefahr zu bannen, dass die Überschussländer die akkumulierten Gläubigerrisiken als Verluste ihrer Banken, Sparer und Steuerzahler abschreiben müssten.

Das derzeitige Programm zur Bewältigung der zweiten Aufgabe entspricht zwar der deutschen Interessenlage, aber es hat auch eine plausible ökonomische Logik für sich: Wenn die Finanzkrisen der GIPS-Staaten die Folge von Leistungsbilanzdefiziten waren, und wenn diese nicht mehr mit den Mitteln der Geld- und Währungspolitik korrigiert werden können, dann müssen fiskalische Austerität und angebotsseitige Strukturpolitik zur Stabilisierung eingesetzt werden. Dieser Logik entsprechen auch die im Dezember 2011 für alle Eurostaaten verschärften Haushaltskontrollen und das neue Sixpack-Verfahren zur Verhinderung makroökonomischer Ungleichgewichte, deren effektive Durchsetzbarkeit freilich noch nicht gesichert erscheint.

Gegenüber den unmittelbar von der Eurokrise betroffenen Ländern jedoch gab es keine Durchsetzungsprobleme. Hier wurden die zur Abwendung des Staatsbankrotts nötigen Kredit-Tranchen nur von Fall zu Fall und jeweils unter präzisen „Konditionalitäten“ ausbezahlt, die von der Kommission definiert und in der Umsetzung von der Troika kontrolliert wurden. Auch dabei ging es um die Kombination von fiskalischer Austerität und angebotsseitigen Strukturreformen. Einerseits mussten staatliche Leistungen beschränkt, Personal entlassen und Verbrauchsteuern erhöht werden, und andererseits sollten Reformen in der Sozialpolitik und im Arbeitsrecht die Löhne senken und so durch „interne Abwertung“ die internationale Wettbewerbsfähigkeit verbessern.

Für die Krisenländer werden jetzt auch die ersten Erfolge vermeldet: Zwar ist die Wirtschaftsleistung gesunken und die Staatsverschuldung deshalb noch gestiegen, aber die Defizite in den Leistungsbilanzen sind deutlich zurückgegangen und die Lohnstückkosten (und damit die überbewerteten realen Wechselkurse) sind ebenfalls gesunken. Allerdings erscheint die Nachhaltigkeit dieser Änderungen noch keineswegs gesichert. Die Verbesserung der Leistungsbilanzen ist weniger einem Anstieg der Exporte geschuldet als dem drastischen Rückgang der Importe wegen der insgesamt schrumpfenden Binnennachfrage. Und die sinkenden Lohnstückkosten sind offenbar eher die Folge hoher Beschäftigungsverluste, wobei vor allem weniger produktive Arbeitsplätze wegfielen. Beide Indikatoren könnten sich also wieder verschlechtern, wenn Nachfrage und Beschäftigung wieder steigen sollten. Mit anderen Worten: Die Euro-Rettungspolitik hat ihr ökonomisches Ziel noch keineswegs erreicht, und jede Entwarnung wäre verfrüht.

Deshalb erscheint es auch nicht verwunderlich, dass die europäische Politik bisher noch nichts zur Bewältigung der dritten Aufgabe unternommen hat. Im Gegenteil: Die rigorosen Sparzwänge haben die Wirtschaft der GIPS-Länder von der Rezession in die Depression getrieben; Entlassungen im öffentlichen Sektor und die Liberalisierung des Arbeitsrechts haben die Arbeitslosigkeit eskalieren lassen; Gehaltskürzungen im öffentlichen Sektor, die Senkung der Sozialleistungen und Mindestlöhne und die Dezentralisierung der Lohnbildung haben die Arbeitseinkommen reduziert; und die Einschränkungen im Gesundheits- und Bildungswesen haben die Folgen steigender Armut weiter verschärft. Im Ergebnis haben die Sparauflagen und die Strukturreformen in manchen Ländern zum Rückgang der Wirtschaftsleistung um mehr als 20\%, zum Anstieg der Jugendarbeitslosigkeit auf mehr als 55\% und zugleich zum radikalen Abbau der sozialen Sicherungssysteme beigetragen. Der Preis der „Euro-Rettung um jeden Preis" sind deshalb tiefe Wirtschafts- und Sozialkrisen, deren Risiken für die gesellschaftliche Integration und die politische Stabilität der Defizitländer von der bisherigen Euro-Rettungspolitik nicht thematisiert, geschweige denn bearbeitet werden.

Eine faire Verteilung der Vorteile und Kosten dieser Rettungsstrategie schließlich stand bisher nicht auf der europäischen Tagesordnung, weil es der deutschen Politik gelang, die von den Strukturen der Währungsunion verursachte Krise als Folge des fiskalischen Fehlverhaltens der Defizitländer zu deklarieren, und weil deren einseitige Abhängigkeit von Rettungskrediten eine extrem asymmetrische Verhandlungssituation im Rat geschaffen hatte, in der diese falsche Situationsdeutung ohne wirksamen Widerstand durchgesetzt werden konnte. 


\section{Solidarhaftung und Transferunion}

Auf die Wirtschafts- und Sozialkrisen der Defizitländer und die Bevorzugung der Gläubiger- und Exportinteressen der Überschussländer konzentriert sich vor allem im Ausland die Kritik an der bisherigen Euro-Rettungspolitik, deren „alternativlose“ Härte man zumeist der asymmetrischen Verhandlungsmacht der deutschen Regierung zuschreibt. Diese Kritik greift jedoch zu kurz, wenn sie lediglich die „harten Konditionalitäten“ angreift. Würden die Sparzwänge gelockert, so stiegen mit den Staatsschulden und der Nachfrage auch die Leistungsbilanzdefizite wieder - und damit die Abhängigkeit von den Kapitalmärkten und das Risiko von Staatsinsolvenzen. Und würden die Reformzwänge gemildert, so schwände mit dem Druck auf die Lohnstückkosten auch die Aussicht auf eine weitere Verbesserung der internationalen Wettbewerbsfähigkeit.

Ein auf Überwindung der akuten Krisen gerichtetes Alternativprogramm müsste viel weiter gehen und sich im Prinzip am Modell der deutschen Vereinigung orientieren. Auch damals hatte die Währungsunion zwischen wirtschaftlich heterogenen Regionen einen extrem verzerrten realen Wechselkurs, ökonomischen Verfall und horrende Massenarbeitslosigkeit in den neuen Bundesländern zur Folge. Die westdeutsche Politik setzte jedoch weder auf fiskalische Austerität noch auf interne Abwertung (die neoliberale Ökonomen auch damals gefordert hatten). Stattdessen folgten auf die deutsche Währungsunion die Sozialunion und die Transferunion. Die neuen Länder wurden in das westdeutsche System der sozialen Sicherung und die westdeutsche Finanzverfassung integriert, und sie verfügen heute über eine moderne öffentliche Infrastruktur, und in manchen Regionen auch über wachstumskräftige Wirtschaftsstrukturen. Aber obwohl die West-Ost-Transfers etwa 3\% des westdeutschen BIP ausmachten, und trotz massenhafter Abwanderung erreicht das BIP pro Kopf im Osten auch heute erst zwei Drittel des deutschen Durchschnitts.

Obwohl Transfers dieser Größenordnung in der Eurozone kaum zu erreichen wären, müssten linke Parteien, die auch von den Verlierern in den Krisenländern der Eurozone gewählt werden wollen, ihr Programm doch an der solidarischen Logik der deutsch-deutschen Währungsunion orientieren. Dabei ginge es nicht nur um Eurobonds zur Verminderung der Zinslast der Staatsschulden, sondern auch um die Verminderung der Kreditabhängigkeit durch Transfers zur Stabilisierung der Sozialsysteme und der öffentlichen Infrastruktur sowie um europäische Investitionsprogramme zur Förderung des Wachstums. Wenn dabei freilich nicht auch gelänge, die Unterschiede der
Wirtschafts- und Lohnbildungsstrukturen zwischen den früheren Hart- und Weichwährungsländern zu überwinden, so könnte die solidarische Option eine langfristige Abhängigkeit der Empfängerländer von externen Transfers begründen.

\section{Demokratisches Dilemma der Europolitik}

Wenn man also die Möglichkeit einer Auflösung der Währungsunion außer Betracht lässt, so müsste eine offene und europaweite parteipolitische Auseinandersetzung über die Bewältigung der Folgen der Eurokrise die Wähler mit zwei riskanten Optionen konfrontieren, die sich in den Folgeproblemen und ihrer Verteilungswirkung radikal unterscheiden:

- Die derzeitige Euro-Rettungspolitik will durch fiskalische Austerität und interne Abwertung die Leistungsbilanzen der Defizitländer ausgleichen und deren internationale Wettbewerbsfähigkeit stärken. Sie nahm und nimmt dafür eine lange Wirtschaftskrise, den extremen Anstieg von Arbeitslosigkeit und Armut und das Risiko sozialer und politischer Desintegration in Kauf. Zugleich schützt sie einseitig die Gläubiger- und Exportinteressen der Überschussländer.

- Das Alternativprogramm einer europäischen Transferunion könnte die soziale Krise mildern, die Binnennachfrage stärken, Investitionen fördern und auch die Arbeitslosigkeit senken. Es nähme dafür neue Leistungsbilanzdefizite und das Risiko stagnierender Wettbewerbsfähigkeit in Kauf - und damit das Risiko einer langfristigen Abhängigkeit von europäischen Transfers, deren Kosten von den Steuerzahlern der Überschussländer getragen werden müssten.

Es gibt also in der Eurokrise keine guten Lösungen, sondern nur die Wahl zwischen komplementär unbefriedigenden Optionen mit gegensätzlichen Verteilungseffekten. Und daraus folgt schließlich das Demokratieproblem der Euro-Rettung: Die gegenwärtige Euro-Rettungspolitik wird unter institutionellen Bedingungen beschlossen, die eine input-orientierte demokratische Legitimation ausschließen. Diese Bedingungen könnten verändert werden, wenn die Europawahlen als Konkurrenz um die Position eines parlamentarisch verantwortlichen Kommissionspräsidenten inszeniert werden und wenn in einem europaweiten Wahlkampf die Parteien gezwungen wären, sich zu den prinzipiell wählbaren Optionen und Folgeproblemen der Europolitik zu erklären. Dann würden die Medien schon dafür sorgen, dass ihre in einem Land vertretenen Positionen auch die Wähler in den anderen erreichen. 
Weil in einem solchen Wahlkampf eher die Verlierer mobilisiert werden, würde die Politisierung der Europolitik die prinzipiell europakritischen Parteien begünstigen. Aber auch die pro-europäischen Parteien könnten, wenn sie in allen Ländern der Eurozone Stimmen gewinnen wollen, die Verteilungskonflikte der Euro-Optionen nicht länger ignorieren. Dann aber ginge es nicht mehr nur um rechts gegen links, Markt-Liberale gegen Sozialdemokraten oder Kapitalbesitzer gegen Arbeitnehmer, sondern es stünden auch die Interessen der deutschen Sparer gegen die der spanischen und irischen Schuldner, und die der Lohnsteuerzahler in den Überschussländern gegen die der Arbeitslosen und Sozialklienten in den Krisenländern. Kurz, soziale und transnationale Verteilungskonflikte würden die politische Auseinandersetzung im Wahlkampf beherrschen.

Aus einem solchen Wahlkampf könnte gewiss kein europaweit legitimierender Konsens über die künftige Richtung der Euro-Rettungspolitik hervorgehen. Das wahrscheinlichste Ergebnis wäre eine Kumulation von Veto-Blöcken, die sowohl die Fortsetzung der bisherigen Austeritäts- und Reformzwangpolitik als auch den Weg zu einer europäischen Transferunion blockieren würden. Noch problematischer wäre es jedoch, wenn dabei eine eindeutige Mehrheit entweder für eine NordOst-Koalition oder für eine Süd-West-Koalition herauskäme.

Weder die EU noch die Eurozone haben ja bisher eine politisch belastbare und legitimationskräftige kollektive Identität (Lincolns "government of the people“) ausbilden können. Zwar wissen die Euro-Völker heute viel mehr übereinander, als man vor wenigen Jahren noch für möglich gehalten hätte. Aber was sie übereinander gelernt haben, ist weniger von wechselseitiger Empathie und Solidarität als von Misstrauen und wechselseitigen Schuldzuweisungen geprägt. Angesichts der massiven Verteilungskonflikte jeder Lösung müssten deshalb die jeweiligen Verlierer der Wahl das von der Mehrheit durchgesetzte Ergebnis nicht als demokratische Legitimation akzeptieren. Und der daraus folgende Verfassungskonflikt könnte die europäische Integration selbst in Frage stellen.

\section{Fazit}

Für die Eurokrise gibt es keine insgesamt guten Lösungen, und die verfügbaren Optionen sind nicht nur jeweils mit gravierenden Nachteilen verbunden, sondern auch mit extrem ungleichen Verteilungsfolgen innerhalb und zwischen den Mitgliedstaaten der Währungsunion. Zugleich fehlt dem derzeit praktizierten autoritären Euro-Regime jede input-orientierte demokratische Legitimation.

Aber so wenig aus der Perspektive einer normativen Demokratietheorie für die Fortsetzung dieses Regimes spricht, so riskant erscheint doch das Vorhaben, es über die Politisierung der Europawahlen stürzen zu wollen. Die Eurokrise hat auch für die pro-europäischen Demokraten ein Dilemma erzeugt, in dem der Versuch, die Krise selbst und die Optionen ihrer Bewältigung zum Gegenstand öffentlicher, demokratischer Auseinandersetzung zu machen, die Chancen des Zusammenwachsens der europäischen Völker nachhaltig beschädigen könnte.

Title: The Democratic Dilemma of Policies to Rescue the Euro

Abstract: European policies imposed in the euro crisis have disabled democratic policy choices at the national level, while the present European euro-rescuing regime lacks democratic legitimacy. But policy choices might now become politicised in the Europe-wide competition of partisan candidates for the Presidency of the European Commission. In that case, voters might indeed be mobilised for or against radically opposed policy options - the continuation of the present austerity regime and the move to a transfer union. The risk is, however, that the escalation of transnational conflict might further divide rather than democratise Europe.

JEL Classification: K10, P00, E42 